Case Report

\title{
Psychiatric Disorders in Möbius Sequence: Intermittent Presentation of Auto-Aggressive Outbursts
}

Vincenzo Prisco ${ }^{1, *}$, Bernadette Donnarumma ${ }^{2}$, Lorenzo Prisco ${ }^{3}$

1. Department of Psychiatry ASL Napoli 1, Naples, Professor at University Federico II, Naples, Italy; E-Mail: vinciprisco@gmail.com

2. Resident in Pediatrics, Department of Pediatrics, University of Naples Federico II, Naples, Italy; EMail: bernadette.donnarumma@gmail.com

3. Emergency medical Service ASL Salerno, Italy; E-mail: Iorenzo.prisco90@gmail.com

* Correspondence: Vincenzo Prisco; E-Mail: vinciprisco@gmail.com

Academic Editor: Laura Orsolini

Special Issue: Recent Advances in Psychiatry

OBM Neurobiology

2020, volume 4, issue 4

doi:10.21926/obm.neurobiol.2004079
Received: March 27, 2020

Accepted: November 19, 2020

Published: December 03, 2020

\begin{abstract}
Anecdotal evidence suggests the possible association of psychiatric disorders with Möbius sequence, although the evidence is not conclusive. Intermittent presentation of autoaggressive outbursts, in particular, could be related to Möbius sequence, different from the common psychotic symptoms. The present report describes the clinical case of a young man with Möbius syndrome who exhibited a complex representation of severe mental retardation and concomitant psychiatric disorders. Extremely rare psychiatric disorders represented the main feature in this clinical case, besides the other well-known signs and symptoms of the Möbius syndrome. Psychiatric disorders could, therefore, prove to be an interesting field of study for an extensive exploration of the Möbius syndrome.
\end{abstract}

\section{Keywords}

Möbius syndrome; autism spectrum disorder; disability; pharmacological treatment

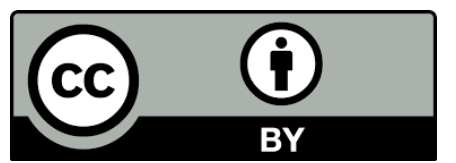

(C) 2020 by the author. This is an open access article distributed under the conditions of the Creative Commons by Attribution License, which permits unrestricted use, distribution, and reproduction in any medium or format, provided the original work is correctly cited. 


\section{Introduction}

Möbius sequence (MBS) is a rare, congenital, non-progressive, uni- or bi-lateral palsy of facial and abducens nerves, with a prevalence of only $0.0002 \%-0.002 \%$ among all births [1]. A clinical diagnosis of MBS is provided when there are congenital cranial nerve VI (abducens) and VII (facial) palsies, often with concomitant malformations in the oro-facial structures, limb anomalies, particularly talipes deformities [2], learning disabilities, and behavioral, emotional, and social difficulties [3]. Moreover, hearing impairment, cleft palate syndrome, and epilepsy are also reported. Even when autism is diagnosed in the patients with MBS2, learning difficulties and high levels of psychopathological maladjustment are uncommon; certain clinical reports suggest a possible sequence variant, presumably more serious, which is characterized by autistic-like symptoms, including alteration in social and interpersonal interaction, difficulties in communication, unusual interests, and behavioral problems, along with an intermittent presentation of auto-aggressive behavior, associated vocalizations, and loss of muscle tone [3].

Autism spectrum disorder (ASD) collectively refers to a group of complex neurodevelopment disorders characterized by repetitive and typical patterns of behavior and difficulties in social communication and interaction. ASD is defined as a condition of altered social and interpersonal interaction, communication difficulties, unusual interests, and behavioral problems.

Interestingly, there are only a few case series that report the psychopathological and personality aspects of the patients with MBS $[4,5]$. These studies concern the depressive symptoms, aggressive behaviors, externalization of problems, thought disorders, and suicidal tendencies. The etiology and pathogenesis of MBS are not yet completely understood. Most of the knowledge regarding MBS has been essentially derived from the related case reports. A possible pathogenesis mechanism of MBS could be the vascular events occurring during the embryonic period. This vascular hypothesis suggests that the ischemic events in the lower brainstem are secondary to the subclavian artery hypoperfusion [6]. Interestingly, abuse of benzodiazepines during pregnancy is also reported to be associated with MBS [7]. Other teratogens associated with MBS are alcohol abuse, electric shock [8], chorionic villus sampling [9], gestational hyperthermia [10], cocaine consumption during pregnancy [11], intake of thalidomide [12], ergotamine [13], or misoprostol [14], and antepartum hemorrhage [15]. The genetic implications in the etiology of MBS are linked with the PLXND1 and REV3L genes, which are reported to be involved in the neural migration control, DNA repair, and disruption of the facial nerve nucleus in mouse models [16]. A de novo autosomal-dominant inheritance is also proposed as a possible mechanism [17].

\section{Materials and Methods}

The present report describes the clinical case of a 35-year-old Caucasian male affected by MBS. Informed consent to participate in the present study was provided by the patient and his caregivers. The case information was collected from the parental report. The syndrome was diagnosed during childhood. The symptoms included severe mental retardation, "mask-like face", incomplete closure of the eyelids during sleep, salivating due to bilateral facial palsy, craniofacial malformation, flattened nasal bridge, and micrognathia. He could consume only a liquid diet due to severe 
dysphagia. Childhood speech problems were detected suddenly, in addition to dysarthria and a global development delay, which are infrequent in MBS as reported in recent studies [2]. He also exhibited early autistic traits, including the impairment of social interaction, communication, and behavior, requiring constant pediatric and neuropsychiatric supervision.

Psychiatric assessment, in particular, gathered the elements of a long history of physical aggression, impulsive anger outbursts, inappropriate sexual behaviors, persecutory and reference delusions, and auditory hallucinations (commenting voices, with a hostile tone). Interestingly, an intermittent expression of auto-aggressive behavior was noted over time. The caregivers of the patient observed an intermittent recurrence of the auto-aggressive behavior, nearly every month, besides REM-sleep behavior disorder with brief crying, vocalizations, and arm/ leg movements during sleep. The patient achieved significantly low results in the Wechsler Adult Intelligence Scale (WAIS), with a QI of 24. He also underwent EEG and overnight polysomnography, and the latter in particular revealed brief episodes of flailing movements of the legs, respiratory effort, and oxygen saturation remaining normal during the night registration. The EEG registered widespread slowing waves. The MRI of the brain, with axial T1 weighted, T2 weighted, and fluid-attenuated inversion recovery (FLAIR) sequences, was normal. The blood test results were within the normal ranges. The patient received treatment with mood stabilizers (sodium valproate), benzodiazepines (delorazepam), and first-generation (haloperidol and zuclopenthixol) or second-generation antipsychotics (olanzapine, risperidone, and aripiprazole), at therapeutic doses, for several years. However, he reported only occasional ameliorations, usually followed by worsened clinical picture, with a clear tendency to the chronic course and a significant social, relational, and occupational dysfunction. Several hospitalizations were required over the years to reduce psychotic symptoms and aggressive behavior. Several EEGs were performed over time, which revealed no other focal neurological sign beside the widespread slowing. The patient could consume only oro-dispersible tablets and solutions due to severe dysphagia.

\section{Discussion}

A pediatrician, a neurologist, and a psychiatrist were in charge of investigating the clinical manifestations of the patient.

Vital signs, including body temperature, were unremarkable. The hepatitis profile, urine analysis, thyroid/liver/kidney function tests, inflammatory indices, serum electrolytes, chest x-ray, and fullabdomen echography were within the normal limits. The other disorders characterized by limb anomalies and congenital facial palsy, which could have caused diagnostic confusion, were excluded. An example of this could be the Poland syndrome, a rare congenital birth defect characterized by hand abnormalities, commonly, including an underdeveloped hand with abnormally short fingers (brachydactyly) or webbed/conjoined digits [fingers or toes] (syndactyly). Autism spectrum disorders lack the physical differences, characteristics of MBS, the facial and abducens nerve palsies, the malformations in the oro-facial structures, and limb anomalies. Mental retardation does not always occur in MBS and is estimated to occur only in $10 \%-15 \%$ of the affected patients, mild in most cases [18]. In the absence of mental disability, the prognosis is poorer.

The patient in the case report received therapy with aripiprazole (15-20 mg/day), sodium valproate solution (2000 mg/day), and promazine (50-100 mg/day) to treat the physical aggression, anger outbursts, inappropriate sexual behaviors, and the auditory hallucinations. Although the 
therapy brought improvement in the psychotic symptoms, the intermittent auto-aggressive behaviors of the patient persisted. Clinical efficacy in the psychotic domains was assessed on the 18item Brief Psychiatric Rating Scale (BPRS) [19] prior to the initiation of the aripiprazole treatment, after which the assessment was performed every week for the complete treatment duration. Clinical response was defined as a minimum of $20 \%$ improvement in the BPRS global score.

This case report described a highly-complex multi-symptomatic subject having MBS and concurrent severe psychiatric symptoms. As opposed to the findings of the previous reports stating that autistic traits (altered social and interpersonal interaction, communication difficulties, unusual interests, and behavioral problems), learning difficulties, and high levels of psychopathological maladjustment are uncommon in the patients with MBS, our clinical case suggests a possible sequence variant, presumably more serious than the previously reported ones. Dawson [20] has reported another possible variant of MBS, which presents with poor impulse control, exhibitionism, and aggression. Other case studies describing psychopathological disorders in association with MBS have referred only to low self-esteem and depressed mood, which results in problematic social interactions, due to the physical abnormalities of MBS4. In our clinical case, an intermittent presentation of auto-aggressive behavior was observed, along with related vocalizations and loss of muscle tone. An accurate anamnesis did not reveal any precipitating stimulus prior to the onset of the aberrant behavior.

There is a possibility of a correlation between REM-sleep behavior disorder and the intermittent presentation of auto-aggressive behavior, which should be further evaluated in future investigations.

Johansson $\mathrm{M}$ et al. [3] indicated a link between brainstem dysfunction and autism, proposed similar pathogenesis for MBS, and identified maternal disorders during pregnancy as a possible causative event for autism spectrum disorders. Such disorders, detected particularly during the firstor mid-trimester of pregnancy, were specifically a result of benzodiazepine abuse, fever, urinary tract infections, epileptic seizures, and maternal uterine bleeding.

It may be hypothesized that the autism spectrum disorders and the MBS share a common pathogenesis. Verzijl1 proposed MBS as a rhombencephalic maldevelopment disorder, resulting from the hypoplasticity of either the corticospinal or the cortico-bulbo-cerebellar long tracts.

\section{Pharmacological Treatment}

According to a previous case report, the medication treatments with clonazepam, melatonin, carbamazepine, sertraline, and temazepam [21] are ineffective.

Moreover, only minimal success is achieved with clomipramine, thioridazine, and carbamazepine, as reported by Dawson [20]. Therefore, these medications may be prescribed to reduce impulsivity, aggression, and inappropriate sexual behaviors.

\section{Outcomes}

The present report emphasized the complexity of managing the severe psychiatric symptoms observed in this case, particularly the ones associated with unexpected outbursts, without prior warning signs, as described in our case.

Interestingly, in our case, the psychotic symptoms improved over time, with continuing intermittent presentation of auto-aggressive behavior, the cause of which remains unknown. There was an improvement in the auditory hallucinations and the persecutory and reference delusions 
upon treatment with medication, although the intermittent auto-aggressive behavior did not appear to respond to the pharmacological treatment. This kind of symptomatology is also reported in episodic dyscontrol syndrome (DSM IV) and Intermittent Explosive Disorder (DSM 5). However, to the best of our knowledge, it has never been previously described for a polymorphic syndrome such as MBS.

The concurrent anger outbursts suggested different pathogeneses, one of which could be the MBS-related parental stress, as described in several previous case reports. It may be hypothesized that a brain alteration undetectable in the MRI may have caused epileptic seizures, which manifested as intermittent aggressive behaviors [22]. The management of such a polymorphic syndrome is difficult, mainly because of the delayed diagnosis, as this disorder remains widely unrecognized among the clinicians.

Multidisciplinary care and a broader understanding of all the clinical presentations of the Möbius syndrome would enable the clinicians to administer adequate treatment to the patient and thereby reduce parental distress.

Nonetheless, the findings described in the present report remain speculative and warrant verification through further investigations conducted in large populations and under a controlled environment.

\section{Conclusions}

1. The symptoms of aberrant behavior and psychiatric disorders should be considered thoroughly by the clinician in charge of treating the Möbius sequence.

2. An intermittent presentation of auto-aggressive behavior could be one of the manifestations of MBS.

3. A multidisciplinary approach is the only way to improve the treatment outcomes of the patient and reduce parental distress.

Further research is required to clarify the real incidence of psychiatric comorbidities and the intermittent presentation of auto-aggressive behavior in MBS.

\section{Competing Interests}

The authors certify that there is no conflict of interest with any financial organization regarding the material discussed in the manuscript

\section{Author Contributions}

Vincenzo Prisco, MD, PhD performed the structure of the study, in particular introduction, material and methods section, discussion and conclusion of the case report; Lorenzo Prisco, MD performed reference section; Bernadette Donnarumma, MD performed language editing.

\section{References}

1. Verzijl HT, van der Zwaag B, Cruysberg JR, Padberg GW. Möbius syndrome redefined: A syndrome of rhombencephalic maldevelopment. Neurology. 2003; 61: 327-333.

2. McKay HV, Touil LL, Jenkins D, Fattah AY. Managing the child with a diagnosis of Moebius Syndrome: More than meets the eye. Arch Dis Child. 2016; 101: 843-846. 
3. Johansson M, Wentz E, Fernell E, Strömland K, Miller MT, Gillber, C. Autistic spectrum disorders in Möbius sequence: A comprehensive study of 25 individuals. Dev Med Child Neurol. 2001; 43: 338-345.

4. Briegel W. Psychopathology and personality aspects of adults with Möbius sequence. Clin Genet. 2007; 71: 376-377.

5. Briegel W, Hofmann C, Schwab KO. Behaviour problems of patients with Moebius sequence and parental stress. J Paediatr Child Health. 2010; 46: 144-148.

6. Bavinck JN, Weaver DD, Opitz JM, Reynolds JF. Subclavian artery disruption sequence: Hypothesis of a vascular etiology for Poland, Klippel-Feil, and Möbius anomalies. Am J Med Genet. 1986; 23: 903-918.

7. Courtens $W$, Vamos $E$, Hainaut $M$, Vergauwen $P$, Laegreid LM. Moebius syndrome in an infant exposed to benzodiazepines. J Pediatr. 1992; 121: 833-834.

8. Martinez-Frias ML, Garcia Mazario MJ, Caldas CF, Conejero Gallego MP, Bermejo E, RodriguezPinilla E. High maternal fever during gestation and severe congenital limb disruptions. Am J Med Genet. 2001; 98: 201-203.

9. Firth HV, Boyd PA, Chamberlain RH, Huson SM, Chamberlain P, Mackenzie IZ. Severe limb abnormalities after chorionic villus sampling at 56-66 days' gestation. Lancet. 1991; 338: 762763.

10. Ghabrial R, Versace P, Kourt G, Lipson A, Martin F. Möbius syndrome: Features and etiology. J Pediatr Ophthalmol Strabismus. 1998; 35: 304-311.

11. Kankirawatana $P$, Tennison MB, D'Cruz O, Greenwood RS. Möbius syndrome in infant exposed to cocaine in utero. Pediatr Neurol. 1993; 9: 71-72.

12. Elsahy NI. Mörbius Syndrome associated with the mother taking thalidomide during gestation. Plast Reconstr Surg. 1973; 51: 93-95.

13. Graf WD, Shephard TH. Uterine contraction in the development of Möbius syndrome. J Child Neurol. 1997; 12: 225-227.

14. Vargas FR, Schuler-Faccini L, Brunoni D, Kim C, Meloni VF, Sugayama SM, et al. Prenatal exposure to misoprostol and vascular disruption defects- a case-control study. Am J Med Genet. 2000; 95: 302-306.

15. Borlot F, da Paz JA, Gonzalez CH, Lucato LT, Marques-Dias MJ. Möbius sequence in a girl and arthrogryposis in her half-brother: Distinct phenotypes caused by prenatal injuries. Fetal Pediatr Pathol. 2011; 30: 260-265.

16. Tomas-Roca L, Tsaalbi-Shtylik A, Jansen JG, Singh MK, Epstein JA, Altunoglu U. De novo mutations in PLXND1 and REV3L cause Möbius syndrome. Nat commun. 2015; 6: 7199.

17. Krueger KE, Friedrich D. Familial congenital disorders of motility of the eye. Klin Monbl Augenheilkd. 1963; 142: 101-117.

18. Kuklik M. Poland-Möbius syndrome and disruption spectrum affecting the face and extremities: A review paper and presentation of five cases. Acta Chir Plast. 2000; 42: 95-103.

19. Overall JE, Gorham DR. The brief psychiatric rating scale. Psychol Rep. 1962; 10: 799-812.

20. Hedges DW, Jeppson KG, Burns C. Twenty-year behavioral follow-up of a 1;13 chromosomal translocation and Mobius syndrome presenting with poor impulse control, exhibitionism, and aggression. Compr Psychiatry. 2009; 44: 462-465.

21. Anderson K, Shneerson J, Smith I. Möbius syndrome in association with the REM sleep behavior disorder. J Neurol Neurosur Ps. 2007; 78: 659-660. 
22. Santos G, Pereira S, Machado O, Sales F, Robalo C, Machado E. Möbius syndrome and epilepsy. J Neuroradiol. 2010; 37: 305-307.

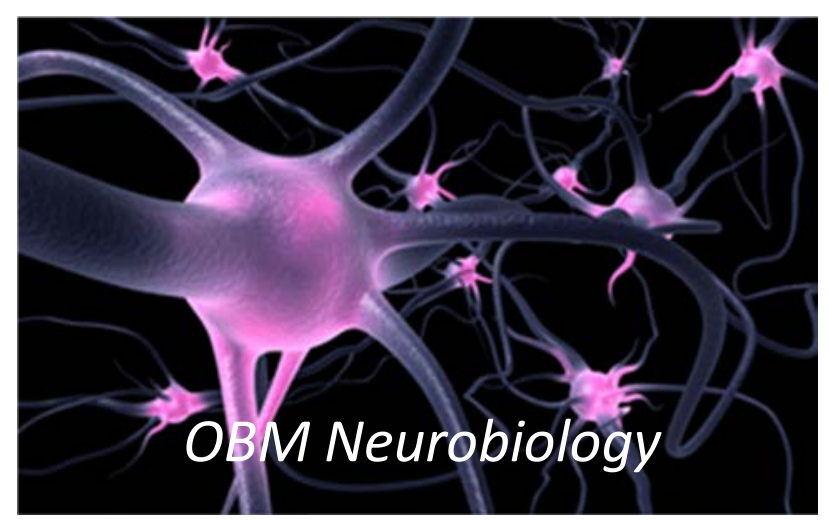

Enjoy OBM Neurobiology by:

1. Submitting a manuscript

2. Joining volunteer reviewer bank

3. Joining Editorial Board

4. Guest editing a special issue

For more details, please visit:

http://www.lidsen.com/journals/neurobiology 